\title{
The Binding and Cleavage Characteristics of Human Hageman Factor during Contact Activation
}

\author{
A COMPARISON OF NORMAL PLASMA WITH PLASMAS DEFICIENT IN \\ FACTOR XI, PREKALLIKREIN, OR HIGH MOLECULAR WEIGHT KININOGEN
}

\author{
Susan D. Revak, Charles G. Cochrane, and John H. Griffin \\ From the Department of Immunopathology, Scripps Clinic and Research Foundation, La Jolla, \\ California 92037
}

\begin{abstract}
A B S T RA C T The ability of human Hageman factor (coagulation factor XII) to bind to a glass surface and its susceptibility to limited proteolytic cleavage during the contact activation of plasma have been studied using normal human plasma and plasmas genetically deficient in factor XI, prekallikrein, or high molecular weight kininogen (HMWK). When diluted normal plasma containing ${ }^{125}$ I-Hageman factor was exposed to a glass surface for varying times, the Hageman factor was found to bind to the surface, and within 5 min became maximally cleaved from its native 80,000 mol wt to yield fragments of 52,000 and $28,000 \mathrm{~mol}$ wt. Hageman factor in factor XI-deficient plasma behaved similarly. In prekallikrein-deficient plasma, the binding of Hageman factor to the glass surface occurred at the same rate as in normal plasma but the cleavage was significantly slower, and did not reach maximum until 60 min of incubation. Cleavage of Hageman factor in HMWK-deficient plasma occurred at an even slower rate, with greater than 110 min of incubation required for maximal cleavage, although the rate of binding to the glass was again the same as in normal plasma. Normal rates of cleavage of Hageman factor were observed for the deficient plasmas after reconstitution with purified human prekallikrein or HMWK, respectively. These observations suggest that normal contact activation in plasma is associated with proteolytic activation of surfacebound Hageman factor.
\end{abstract}

Portions of this work have appeared in abstract form (1976. Fed. Proc. 35: 692).

Dr. Griffin is the recipient of a Research Career and Development Award, HL-00192.

Received for publication 28 October 1976 and in revised form 31 January 1977.
The cleavage of the surface-bound Hageman factor molecule responsible for the formation of the 52,000and 28,000-mol wt fragments occurred at two closely situated sites, one of which was within a disulfide loop. Cleavage at the site external to the disulfide bond resulted in the release from the surface of the 28,000-mol wt fragment. Cleavage at the site within the disulfide loop resulted in the formation of a 28,000 mol wt fragment which remained surface bound, presumably by virtue of the disulfide linkage to the larger fragment.

\section{INTRODUCTION}

When normal human plasma is exposed to glass, a contact activation process takes place, leading ultimately to coagulation, kinin formation, and fibrinolysis. Central to all three systems is the activation of Hageman factor. Studies presented in recent years have shown the importance of prekallikrein and high molecular weight kininogen $(\mathrm{HMWK})^{1}$ in the activation of Hageman factor. The participation of kallikrein in coagulation was suggested in 1972, when it was shown that the addition of purified rabbit or human kallikrein to rabbit plasma promoted its clotting (1). Later studies, using purified human plasma enzymes, established that kallikrein acted as a proteolytic activator of Hageman factor $(2,3)$ and suggested that it was 5-10 times more active on a molar basis than the other plasma enzymes (plasmin and activated factor XI) found capable of activating Hageman factor

\footnotetext{
${ }^{1}$ Abbreviations used in this paper: HMWK, high molecular weight kininogen; PBS, phosphate-buffered saline $(0.01 \mathrm{M}$ sodium phosphate, $0.15 \mathrm{M} \mathrm{NaCl}, \mathrm{pH} 7.0$ ); SDS, sodium dodecyl sulfate.
} 
(4). Cleavage of the Hageman factor molecule in solution was found to occur concomitantly with its activation by kallikrein (5). The potential for a reciprocal activation process in plasma involving Hageman factor and prekallikrein was thus established. Confirmation of this hypothesis occurred when a deficiency in prekallikrein (6-9) was found to be responsible for the abnormal clotting, fibrinolytic, and kinin-generating activities of a plasma known as Fletcher trait plasma $(10,11)$.

The necessity of an additional protein for the efficient activation of factor XI by Hageman factor was first reported by Schiffman and Lee who termed the protein "contact activation cofactor" (12). Again, however, it was the identification of a specific protein, in this instance HMWK (13-16), missing in another group of plasmas showing abnormal clotting, fibrinolytic, and kinin-generating activities (13-19) which unequivocably established the importance of the protein in the contact activation of Hageman factor. Later studies suggested that contact activation cofactor was HMWK (19). Studies in our laboratory employing purified Hageman factor, prekallikrein, factor XI, and HMWK have shown that HMWK accelerates by at least 10-fold the activation of factor XI and prekallikrein by surface-bound active Hageman factor as well as the cleavage of surface-bound Hageman factor by kallikrein $(20,21)$. Meier et al. have also reported that HMWK enhances the expression of the activity of surface-bound Hageman factor (22). A requirement for HMWK in the activation of factor XI by Hageman factor and ellagic acid has also been shown (23), and a two- to threefold increase in the activation of prekallikrein by active Hageman factor fragments in solution has been reported (24).

It thus appears that effective contact activation of Hageman factor in plasma requires prekallikrein and HMWK, and that plasmas deficient in either of these proteins do not develop normal clotting, kinin, and fibrinolytic activities upon exposure to glass. But the effect of these proteins on the Hageman factor molecule in plasma during contact activation is unknown. We therefore undertook studies to determine what happens structurally to the Hageman factor molecules in plasma when a glass surface is introduced. A comparison of the results obtained in normal plasma with those obtained using prekallikrein-deficient and HMWK-deficient plasmas enhanced our understanding of the molecular events involved in effective contact activation.

\section{METHODS}

Plasmas. The normal human plasma used in these studies was from a pool of 19 normal healthy individuals. Blood was collected into one-sixth-volume acid-citrate-dextrose anticoagulant, centrifuged at $3,000 \mathrm{rpm}$ for $20 \mathrm{~min}$ at room temperature, and after separation from the cellular components, recentrifuged at $5,000 \mathrm{rpm}$ for $40 \mathrm{~min}$ at $4^{\circ} \mathrm{C}$. Aliquots were stored at $-70^{\circ} \mathrm{C}$. HMWK-deficient plasma (Fitzgerald) was obtained from Mr. George King (George King Biomedical, Salem, N. H.). It contained $43 \%$ prekallikrein antigen compared to a pool of 19 normal plasmas as determined by radial immunodiffusion, and $14 \%$ normal levels when assayed for procoagulant activity in prekallikrein-deficient plasma. Factor XI-deficient plasma was also obtained from Mr. George King. Prekallikrein-deficient plasma was supplied by Dr. Charles Abildgaard, University of California at Davis, School of Medicine.

Plasma proteins. Hageman factor was purified from pooled normal human plasma as described elsewhere (25). The preparations used were determined to be greater than $95 \%$ homogeneous on polyacrylamide gels run in the presence or absence of sodium dodecyl sulfate(SDS). Clotting assays in Hageman factor-deficient plasma yielded a specific clotting activity of $80 \pm 15 \mathrm{U} / \mathrm{mg}$ protein. No factor XI or prekallikrein activity was detectable $(<0.1 \%)$. Clotting activity was unaltered by the radiolabeling of the protein with ${ }^{125} I$ according to the procedure of McConahey and Dixon (26). Uptake of radiolabel varied between 1 and $9 \mu \mathrm{Ci} / \mu \mathrm{g}$. Distribution of the radiolabel in the molecule also varied and was characterized for each preparation by experiments such as that shown in Fig. 1. The native molecule having a mol wt of 80,000 was consistantly cleaved to yield fragments of 52,000 and $28,000 \mathrm{~mol}$ wt but the relative amount of radiolabel appearing in these two peaks varied with different preparations of ${ }^{125}$ I-Hageman factor. For the experiments investigating the rate of binding of Hageman factor to a surface, a preparation was chosen in which almost all of the radiolabel was in the 52,000 mol wt fragment. The variable distribution of radiolabel did not affect any other experiments since the cleavage of the molecule was assessed by the disappearance of radiolabel from the 80,000 -mol wt peak.

The human prekallikrein employed in the reconstitution experiments was obtained from Dr. Richard Ulevitch and $\mathrm{Mr}$. Alan Johnston of our laboratory and was prepared by them using a procedure based on modifications of previously described methods (27). The major modifications included elimination of the ammonium sulfate precipitation, second DEAE column, and Pevikon electrophoresis (Mercer Consolidated Corp., Yonkers, N.Y.), and the addition of a chromatographic step employing hydroxylapatite. The preparations contained as identifiable contaminants gamma globulin and $\beta_{2}$ glycoprotein I. It contained no detectable plasminogen or factor XI. Small amounts of active kallikrein also present were removed by incubation with insolubilized soybean trypsin inhibitor before use. Quantitation of prekallikrein was based on clotting assays in prekallikrein-deficient plasma, kindly performed by Dr. Bonno Bouma of our laboratory, and is expressed as activity relative to standard normal plasma dilutions. The preparation used had a sp act of $0.85 \mathrm{U} / \mathrm{mg}$ protein.

Human HMWK was purified by a modification of the method of $\mathrm{Habal}$ et al. (28). ${ }^{2}$ The major changes in the procedure as published were the use of DEAE-Sephadex (Pharmacia Fine Chemicals Inc., Piscataway, N. J.) resin instead of QAE-Sephadex, elimination of the G-200 Sephadex column, and the substitution of SP-Sephadex for CM-Sephadex in the final step. The preparations used were found to be greater than $95 \%$ homogeneous as judged by polyacrylamide gel analysis. Quantitation of activity based on

\footnotetext{
${ }^{2}$ Griffin, J. H. Manuscript in preparation.
} 
clotting assays in HMWK-deficient plasma yielded a sp act of 10-11 U/mg protein.

Contact activation of plasma in glass tubes. For the studies reported here, it was necessary to devise an experimental system of contact activation in which rates of binding and cleavage of ${ }^{125} \mathrm{I}$-Hageman factor added to plasma could be quantitatively assessed. Kaolin, often used as an activating surface in coagulation studies, could not be used because the time involved in washing and centrifuging the samples was too long to allow precise end-point determinations for short incubation times. A single glass surface, such as the bottom of a test tube, overcame this problem. To establish conditions to achieve optimal rates of binding and cleavage of Hageman factor in normal plasma added to this "limited" glass surface, preliminary experiments were conducted in which different volumes of varying dilutions of plasma containing ${ }^{125} \mathrm{I}$-Hageman factor were added to glass tubes and were analyzed for binding and cleavage of Hageman factor. Although the system of contact activation described below is optimal for the studies described, it should be noted that the rates of binding and cleavage of Hageman factor obtained using it will differ from the rates of binding, cleavage, or activation obtained using other systems, although relative differences between different plasmas would be similar.

Citrated plasma was diluted 1:6 with phosphate-buffered saline (PBS, 0.01 M sodium phosphate, $0.15 \mathrm{M} \mathrm{NaCl}, \mathrm{pH}$ 7.0) in a plastic tube. 3-6 $\mu \mathrm{l}(0.04-0.08 \mu \mathrm{g})$ of ${ }^{125} \mathrm{I}-\mathrm{H}$ ageman factor was added per $100 \mu \mathrm{l}$ of diluted plasma. This resulted in a 10-20\% increase in Hageman factor concentration in the plasma. Except where noted differently, $20-\mu \mathrm{l}$ samples were added to the bottom of new, unwashed $12 \times 75-\mathrm{mm}$ borosilicate glass tubes and shaken at room temperature for varying times. The reactions were stopped by the addition of $50 \mu \mathrm{l}$ of $4 \% \mathrm{SDS}$ in the presence or absence of $2 \% \beta$-mercaptoethanol as indicated. In some experiments, the supernate (e.g. the fluid content of the tube which would contain all soluble material not bound to the glass surface) was removed from the incubation tube at the end of the incubation period and added to $50 \mu \mathrm{l}$ of SDS in the presence or absence of mercaptoethanol. In these cases, the glass incubation tube was then washed twice with $0.2 \mathrm{ml}$ PBS before receiving $50 \mu \mathrm{l}$ of SDS with or without mercaptoethanol. All samples were placed in a boiling water bath for $3 \mathrm{~min}$. Under these conditions, greater than $95 \%$ of the surface-bound material was eluted from the glass tubes and could then be analyzed by electrophoresis on SDS-polyacrylamide gels. As a control, ${ }^{125} \mathrm{I}-$ Hageman factor added to a 1:6 dilution of normal plasma and incubated in plastic tubes showed no cleavage of Hageman factor occurring after $2 \mathrm{~h}$ of incubation.

SDS-polyacrylamide-gel electrophoresis. Electrophoresis in 5 or $7 \%$ polyacrylamide gels containing $0.1 \%$ SDS was performed according to the method of Weber and Osborn (29) in $4.5 \times 100-\mathrm{mm}$ glass tubes. Samples containing ${ }^{125} \mathrm{I}-$ Hageman factor were prepared as described above. Bromophenol blue was added as a tracking dye and the samples were applied in glycerol to the gels. After $45 \mathrm{~min}$ at $4 \mathrm{~mA} /$ tube, electrophoresis was continued at up to $10 \mathrm{~mA} /$ tube until the tracking dye was within $1 \mathrm{~cm}$ of the end. Gels were sliced into $1.2-\mathrm{mm}$ segments and each slice was counted for radioactivity. Greater than $90 \%$ of the radioactivity applied to the gels was recovered. Molecular weight values were determined by comparison with stained gels of marker proteins run in parallel. The marker proteins used and their molecular weights were: human gamma globulin (unreduced), 150,000; phosphorylase-a, 100,000; trans-
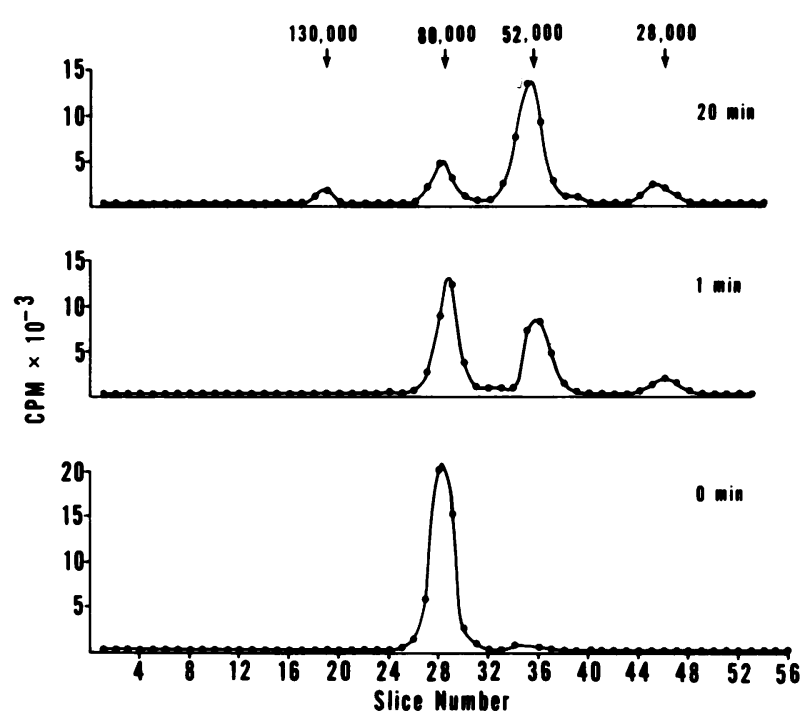

FIGURE 1 Cleavage patterns of Hageman factor upon exposure of plasma to glass. Electrophoresis of the reduced samples was on $5 \%$ polyacrylamide gels containing SDS. The anode is to the right.

ferrin, 90,000; bovine serum albumin, 68,000; chymotrypsinogen, 43,000; ovalbumin, 25,700; and cytochrome-c, 12,000.

\section{RESULTS}

Cleavage pattern of Hageman factor in normal plasma upon exposure to glass. The cleavage of Hageman factor molecules during contact activation of plasma was studied by incubating normal human plasma containing ${ }^{125} \mathrm{I}$-Hageman factor in glass tubes, and observing the pattern of radioactivity obtained by electrophoresis of the activated samples on polyacrylamide gels containing SDS. In these initial studies, $5-\mu$ l samples of diluted normal human plasma were incubated in glass tubes for varying times and the reactions were stopped by the addition of $50 \mu \mathrm{l}$ of a $2 \%$ SDS solution containing $1 \%$ mercaptoethanol. After a 3-min incubation of the sample tube in a boiling water bath, the samples were applied to $5 \%$ polyacrylamide gels containing $0.1 \%$ SDS and the resultant radioactivity patterns were then analyzed. Fig. 1 shows the patterns observed after 0,1 , and 20 min of incubation of plasma in glass tubes. The 0-min sample was obtained by adding the diluted plasma sample directly to the SDS solution. The initial peak at $80,000 \mathrm{~mol}$ wt seen at $0 \mathrm{~min}$ decreases upon incubation and new peaks appear at 52,000, 28,000, and $130,000 \mathrm{~mol} \mathrm{wt}$. The peak at $130,000 \mathrm{~mol} \mathrm{wt}$ is the result of a complex of the fragment normally migrating at $28,000 \mathrm{~mol}$ wt with $\mathrm{Cl}$ esterase inhibitor (30). When incubation is continued for longer time periods, a slow decrease in the $52,000-\mathrm{mol}$ wt peak is 


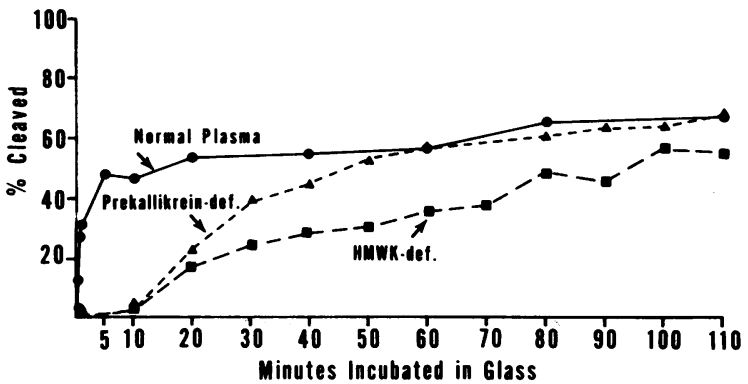

FIGURE 2 Rates of cleavage of Hageman factor in normal, prekallikrein-deficient (def.) and HMWK-deficient (def.) plasmas upon exposure to glass. The percent of Hageman factor cleaved was calculated from the cleavage patterns obtained on SDS gels.

seen and a new peak of $40,000 \mathrm{~mol}$ wt concomitantly appears. These data suggest that Hageman factor in normal plasma, when exposed to a glass tube, is cleaved from its native 80,000 -mol wt form to yield fragments of 52,000 and 28,000 mol wt. Subsequent cleavage of the 52,000 fragment with the appearance of a 40,000-mol wt fragment indicates a secondary cleavage site. As previously reported (5), both sites are equally susceptible to cleavage when purified Hageman factor is incubated with trypsin in solution.

Rate of cleavage of Hageman factor in normal, factor XI-deficient, prekallikrein-deficient, and HMWK-deficient plasmas upon exposure to glass. To compare the rate of cleavage of Hageman factor in normal plasma to its rate of cleavage in plasmas deficient in various proteins, samples containing radiolabeled Hageman factor and diluted plasma were prepared using normal, factor XI-deficient, prekallikrein-deficient, and HMWK-deficient plasmas. 20- $\mu \mathrm{l}$ samples were incubated in glass tubes for varying periods up to $110 \mathrm{~min}$. Mercaptoethanol was added to each sample before analysis on SDS polyacrylamide gels. The percent of the total radioactivity recovered from the gel which appeared at a position corresponding to $80,000 \mathrm{~mol} \mathrm{wt}$ was tabulated for each sample. With the amount of radiolabel appearing at 80,000 mol wt in the 0 -min incubation sample taken as $100 \%$, the percent of Hageman factor remaining at 80,000 mol wt was calculated for each time point. By subtracting this value from $100 \%$, the data were expressed as the percent of native Hageman factor molecules which were cleaved. This is shown in Fig. 2 for normal, prekallikrein-deficient, and HMWKdeficient plasmas as a function of incubation time in glass. Maximal cleavage of Hageman factor was observed in normal plasma by $5 \mathrm{~min}$, after $60 \mathrm{~min}$ in prekallikrein-deficient plasma, and longer than 110 min in HMWK-deficient plasma. The kinetics of cleavage of Hageman factor in factor XI-deficient plasma was the same as in normal plasma (not shown).
Fig. 2 represents the data from one set of experiments. Other experiments yielded very similarly shaped curves with the same relative differences between the various plasmas.

Restoration of normal cleavage of Hageman factor in prekallikrein-deficient and $H M W K$-deficient plasmas by reconstitution of the plasmas with purified prekallikrein and HMWK. To confirm that the abnormally slow rate of cleavage of Hageman factor in prekallikrein-deficient plasma was exclusively due to a lack of this protein, reconstitution experiments were performed in which increasing amounts of normal plasma or partially purified prekallikrein were added to the prekallikrein-deficient plasma. For example, for a $2 \%$ reconstitution with normal plasma, a mixture was made which consisted of $98 \%$ prekallikrein-deficient plasma and $2 \%$ normal plasma. The mixture was then diluted 1:6 with PBS and a trace of ${ }^{125}$ I-Hageman factor was added. For a $2 \%$ reconstitution with prekallikrein, $0.02 \mathrm{U}$ of prekallikrein were added to each milliliter of prekallikrein-deficient plasma, the additional volume being subtracted from the amount of PBS added to obtain the subsequent 1:6 dilution. 20- $\mu$ l samples of the prekallikrein-deficient plasma containing ${ }^{125} \mathrm{I}$-Hageman factor and reconstituted with increasing amounts of either normal plasma or partially purified prekallikrein were incubated in glass tubes for $2 \mathrm{~min}$. After the addition of SDS and mercaptoethanol, the extent of cleavage of Hageman factor was assessed by SDS-polyacrylamide gels. Normal plasma incubated in glass for $2 \mathrm{~min}$ was run as a control and the amount of cleavage of Hageman factor in it was taken as the maximal value at $2 \mathrm{~min}$. Cleavage of Hageman factor in the reconstituted plasma samples, expressed as percent of maximal, is shown in Fig. 3. The addition of $10 \%$ normal plasma raised the level of cleavage to $80 \%$ of normal. Reconstitution with the partially purified protein was found to closely parallel the reconstitution with normal plasma.

Similar experiments were performed reconstituting

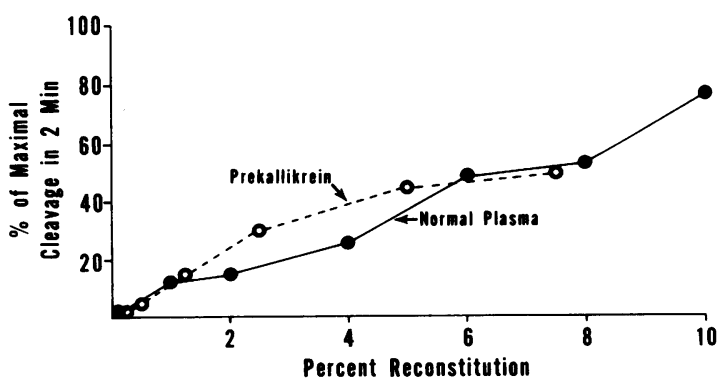

FIGURE 3 Reconstitution of the cleavage of Hageman factor in prekallikrein-deficient plasma by the addition of normal plasma or partially purified prekallikrein. Cleavage of the reconstituted plasmas is expressed as percent of the maximal value observed after 2 -min incubation. 


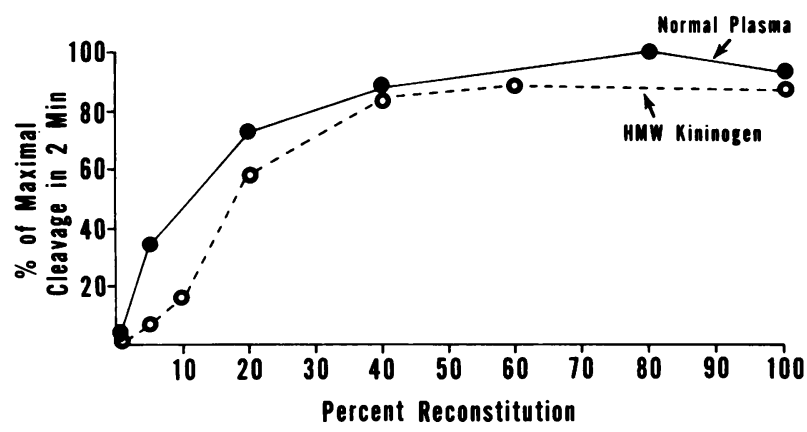

FIGURE 4 Reconstitution of the cleavage of Hageman factor in HMW kininogen-deficient plasma by the addition of normal plasma or purified HMW kininogen. Cleavage of the reconstituted plasmas is expressed as percent of the maximal value of cleavage observed after 2-min incubation.

HMWK-deficient plasma with normal plasma and with highly purified HMWK. The results are shown in Fig. 4. Approximately $40 \%$ reconstitution with normal plasma or HMWK is necessary to reach a level of $80 \%$ normal cleavage.

The influence of the reduction of disulfide bonds on the dissociation of cleaved Hageman factor molecules. To determine whether the Hageman factor fragments produced during contact activation of plasma were covalently linked by disulfide bonds, duplicate 20- $\mu$ l diluted plasma samples containing ${ }^{125}$ I-Hageman factor were subjected to contact activation in glass tubes as described above. At the end of the desired incubation times, one plasma sample received 50 $\mu \mathrm{l}$ of a $4 \%$ SDS solution, whereas the other plasma sample received $50 \mu \mathrm{l}$ of a $4 \%$ SDS solution containing $2 \%$ mercaptoethanol. All samples were incubated for $3 \mathrm{~min}$ in a boiling water bath before electrophoresis on $7 \%$ polyacrylamide gels containing SDS. Cleavage fragments of 52,000, 28,000, and at longer incubation times, $40,000 \mathrm{~mol} \mathrm{wt}$ were observed under both reducing and nonreducing conditions. However, the rate of appearance of the cleavage fragments was significantly slower in the absence of the reducing agent. The same effect of mercaptoethanol treatment on Hageman factor was observed in normal, prekallikrein-deficient, and HMWK-deficient plasmas. The data are shown in Fig. 5. The presence of $8 \mathrm{M}$ urea in the samples which lacked mercaptoethanol did not result in any increase in dissociation of the fragments, thus making less likely the possibility that the cleaved fragments might be noncovalently associated. The data indicate that when $50 \%$ of the Hageman factor molecules have been cleaved, approximately half of them contain disulfide linkages between the resulting fragments.

Location of Hageman factor fragments and residual uncleaved Hageman factor molecules after the incubation of plasma with glass. To determine if the
Hageman factor fragments produced by the incubation of plasma in glass tubes were surface bound, supernates were removed from the glass tubes at the end of the incubation time and added to $50 \mu \mathrm{l}$ of a $4 \%$ SDS solution in the presence or absence of $2 \%$ mercaptoethanol. The glass incubation tube was washed twice with $200 \mu \mathrm{l}$ of PBS. The addition of $50 \mu \mathrm{l}$ of $4 \%$ SDS in the presence or absence of $2 \%$ mercaptoethanol, followed by a 3-min incubation of the tube in a boiling water bath, eluted $95-100 \%$ of the surface-bound material. The supernate and surfacebound samples were then analyzed by SDS-polyacrylamide-gel electrophoresis. Fig. 6 shows the results obtained for a 5-min incubation time. In the sample of the surface-bound material in the absence of mercaptoethanol, radioactivity was found at positions corresponding to 80,000 and $52,000 \mathrm{~mol}$ wt. The 28,000 mol wt fragment appeared in the supernate. When the surface-bound samples were treated with mercaptoethanol, however, no peak was observed at 80,000 mol wt, the peak at $52,000 \mathrm{~mol}$ wt was increased, and a 28,000-mol wt peak appeared. Hageman factor

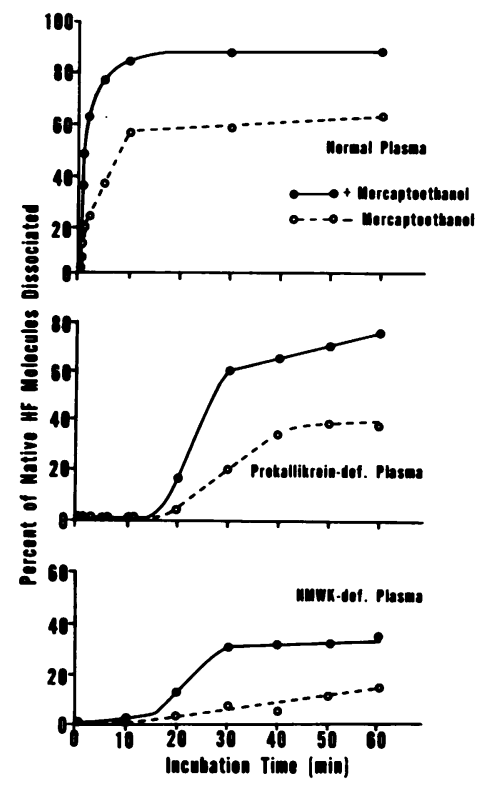

FIGURE 5 The effect of mercaptoethanol on the dissociation of ${ }^{125} \mathrm{I}$-Hageman factor after incubation of plasma in glass. $20-\mu l$ aliquots of 1:6 dilution of normal plasma, prekallikrein-deficient (def.), and HMWK-deficient (def.) plasmas containing ${ }^{125} \mathrm{I}-\mathrm{H}$ ageman factor were incubated in glass tubes at $22^{\circ} \mathrm{C}$. At the indicated times, $50 \mu \mathrm{l}$ of $4 \%$ SDS in the presence $(O)$ or absence $(O)$ of mercaptoethanol was added to stop the reaction and effect the elution of any surfacebound material. After analysis of each sample on SDSpolyacrylamide gels, the percent of ${ }^{125}$ I-Hageman factor migrating at a position corresponding to $80,000 \mathrm{~mol}$ wt was calculated. Data are expressed as the percent of native 80,000 -mol wt Hageman factor (HF) molecules that have been dissociated. 

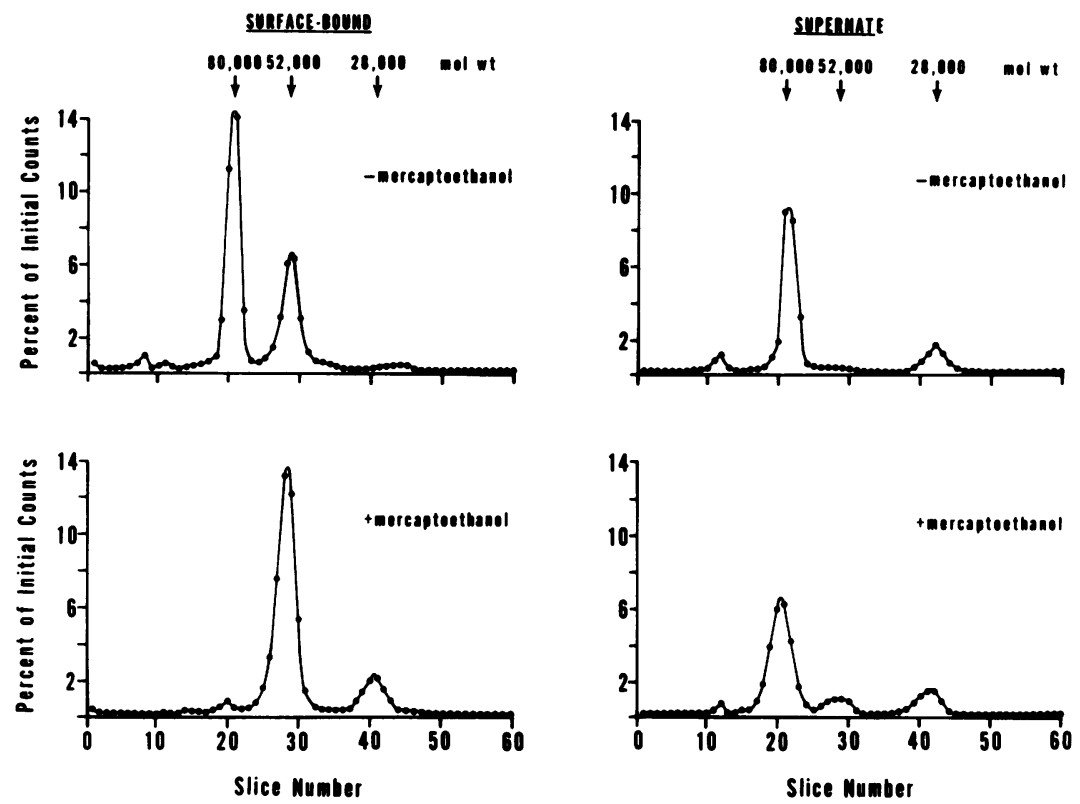

FIGURE 6 Location of ${ }^{125}$ I-Hageman factor fragments after the incubation of plasma with glass. After a 5-min incubation in glass tubes, plasma samples were separated into supernates and surface-bound samples, and each was analyzed in the presence and absence of mercaptoethanol on SDS-polyacrylamide gels. Data are expressed as the percent of initial radioactivity in the incubation tube. The addition of mercaptoethanol to the surfacebound material results in the disappearance of the $80,000-\mathrm{mol}$ wt peak, an increase in the $52,000-\mathrm{mol}$ wt peak, and a new peak at 28,000 mol wt. Reduction had little effect on the supernatant material which contained predominantly 80,000 -mol wt native Hageman factor and 28,000-mol wt fragments.

which retained its native mol wt of 80,000 in the presence of mercaptoethanol was found only in the supernate. These data are consistent with the hypothesis that Hageman factor in plasma becomes bound to the glass surface and proteolytically cleaved to yield fragments of 52,000 and 28,000 mol wt. Approximately one half of the cleaved molecules contain a disulfide linkage between the fragments and remain surface bound. In those molecules in which the fragments are not linked by disulfide bonds, the 52,000 -mol wt fragment remains surface bound whereas the 28,000-mol wt fragment is released into solution. The presence of additional Hageman factor of native molecular weight in the supernate after treatment with mercaptoethanol suggests that there is either a limiting amount of glass surface for the binding of Hageman factor or that further binding is being inhibited.

The rate of binding of Hageman factor to a glass surface in normal, prekallikrein-deficient, and HMWKdeficient plamas. Since binding to the glass surface appeared to be a prerequisite for cleavage of Hageman factor in normal plasma, the possibility that the failure of the prekallikrein-deficient and HMWK-deficient plasmas to exhibit rapid cleavage of Hageman factor might be caused by a lack of binding of Hageman factor to the glass surface was examined. The kinetics of the binding reaction were studied using a preparation of ${ }^{125}$ I-Hageman factor which, fortuitously, contained a minimal amount of radiolabel on the 28,000mol wt fragment. Preliminary experiments had indicated that the 52,000-mol wt fragment remained bound to the surface (Fig. 6). Therefore, the total amount of Hageman factor binding could be easily assayed by removing the supernate at the end of the incubation period, washing the glass tube, and then counting the radioactivity remaining bound to the glass. $20-\mu \mathrm{l} \mathrm{sam-}$ ples of diluted plasma containing ${ }^{125}$ I-Hageman factor were used. Two washes of $0.2 \mathrm{ml}$ PBS were found to be sufficient to remove all unbound radioactivity. Supernate and washes were saved and counted to determine the total radioactivity initially present. The kinetics of binding of Hageman factor to the glass surface in the presence of normal, prekallikreindeficient, and HMWK-deficient plasmas are shown as the solid lines in Fig. 7. The rate of binding of Hageman factor to the glass surface is the same in all three plasmas. The percent of Hageman factor cleaved in each plasma (obtained from experiments performed as described above) is included for comparison in this figure (dashed lines). It should also be noted that in normal plasma (upper panel), the binding of 
Hageman factor to the glass surface precedes by approximately $40 \mathrm{~s}$ the cleavage of the molecules.

\section{DISCUSSION}

Evidence for the rapid proteolytic cleavage of Hageman factor upon the exposure of normal human plasma to glass has been presented. The rate of this cleavage was found to be far slower in plasmas deficient in either prekallikrein or HMWK, whereas factor XI-deficient plasma yielded the same rapid rate of cleavage of Hageman factor as normal plasma. Successful restitution of the normal cleavage rate of Hageman factor in prekallikrein-deficient plasma with partially purified prekallikrein, and of HMWK-deficient plasma with highly purified HMWK, decreases the possibility of the potential influence of other, unknown factors in the deficient plasmas and supports the assertion that prekallikrein and HMWK are the proteins in plasma essential for the rapid cleavage of Hageman factor. Studies describing the abnormalities of clot formation, kinin generation, and fibrinolysis during contact activation of prekallikrein-deficient plasma (8-11) and HMWKdeficient plasma (13-18) have suggested an interaction of these proteins with Hageman factor. Coupled with those previous observations, the data presented here provide strong evidence that the failure of the Hageman factor molecule to become cleaved on the glass surface is coincident with its failure to become activated in these plasmas.

Since binding to the glass surface appeared to be necessary for the rapid cleavage of Hageman factor, the rates of binding were compared in normal, prekallikrein-deficient, and HMWK-deficient plasmas to ascertain whether the failure to exhibit normal cleavage might be associated with abnormal binding in these plasmas. The results, shown in Fig. 7, show no differences in binding rates in the plasmas tested. Therefore, the participation of prekallikrein and HMWK in the cleavage process appears to be subsequent to the binding of the Hageman factor to the surface.

The observed failure of prekallikrein-deficient plasma to produce a normal rate of cleavage of Hageman factor, and data from earlier reports on the potent action of kallikrein on Hageman factor which results in cleavage and activation of the Hageman factor, leads to the conclusion that kallikrein is the enzyme in normal plasma responsible for the rapid cleavage of the Hageman factor molecule. The alternative possibility, that prekallikrein or kallikrein is essential for the generation of some other enzyme which then acts proteolytically on Hageman factor, is considered unlikely. Recent studies using purified reagents have shown that the factor XI-activating capacity of plasma
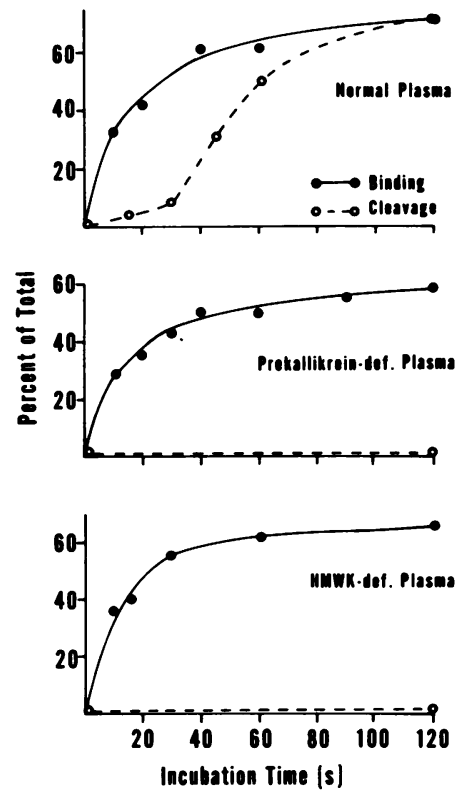

FIGURE 7 Rates of binding and cleavage of Hageman factor in normal, prekallikrein-deficient (def.), and HMWK-deficient (def.) plasmas upon exposure to glass. Binding data are expressed as the mean of at least three determinations per time point.

could be reproduced using Hageman factor, HMWK, and prekallikrein $(20,21)$. However, the slow cleavage observed in prekallikrein-deficient plasma (Fig. 2) does indicate that at least one other enzyme is capable of proteolytic action on Hageman factor in plasma. Although its identity is unknown, plasmin $(2-4,31$, 32 ) and activated factor XI (4) are potential candidates. Less likely is the possibility that trace quantities of prekallikrein might be present in the prekallikreindeficient plasma. This slow cleavage of Hageman factor on glass in prekallikrein-deficient plasma offers a plausible explanation for the slow generation of activity of the contact phase of coagulation in prekallikreindeficient plasma which occurs upon prolonged incubation with glass or kaolin.

The data presented here also indicate a critical role for HMWK in the cleavage of Hageman factor in plasma on a surface. Using purified proteins, it has been shown that HMWK is capable of increasing the rate of cleavage of surface-bound Hageman factor by kallikrein by at least 8-11-fold $(20,21)$. Additionally, HMWK was found capable of a 20 -fold augmentation of the conversion of prekallikrein to kallikrein by surface-bound active Hageman factor $(20,21)$. With the necessity of reciprocal activation of Hageman factor and prekallikrein for effective contact activation in plasma, the role of HMWK as a cofactor in the activation of prekallikrein underscores its importance for a rapid rate of cleavage of Hageman factor. 


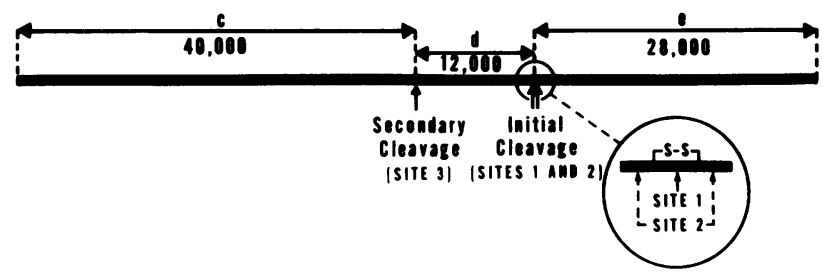

Figure 8 Cleavage of human Hageman factor during contact activation of plasma. The $80,000-\mathrm{mol}$ wt native Hageman factor molecule can be divided into three regions, $c, d$, and e, as described previously (30). When normal plasma is exposed to a glass surface, the Hageman factor molecules are rapidly cleaved at sites 1 and/or 2 . As shown in the enlargement inset, site 1 is within a disulfide loop whereas site 2 is outside of it; the uncertainty of its orientation relative to site 1 being indicated by the two dashed arrows. After prolonged incubation with glass, cleavage at site 3 occurs.

The cleavage of Hageman factor observed during contact activation results in the formation of fragments of 52,000 and $28,000 \mathrm{~mol}$ wt. The observed difference in dissociation of the cleaved Hageman factor molecules in the presence or absence of mercaptoethanol suggests the possibility of two closely spaced cleavage sites on the Hageman factor molecule with the presence of a half-cystine residue between them. The method employed here of analysis by SDSpolyacrylamide-gel electrophoresis is unable to resolve fragments with molecular weights differing by less than 5\%. Fig. 8 shows schematically these two cleavage sites as well as the site responsible for the secondary cleavage of the 52,000-mol wt fragment observed at prolonged incubation times. If cleaved at site 1 , the 28,000-mol wt fragment, designated e fragment, remains linked by a disulfide bridge to the $52,000-\mathrm{mol}$ wt fragment, designated cd fragment; cleavage at site 2 dissociates the $e$ fragment. It has previously been reported that the areas responsible for the binding of the Hageman factor molecule to the surface are located in the $\mathrm{c}$ and $\mathrm{d}$ regions (30). Therefore, if the surface-bound molecule is cleaved only at site 1 , the e fragment would be expected to remain surface associated whereas cleavage at site 2 would release the e fragment into the supernate. The current experiments support this concept.

It is possible that the dual presence of Hageman factor molecules containing a disulfide bond which links the cd fragment and the e fragment, and molecules which lack this bond might be due to the existence of multiple forms of human Hageman factor. Insufficient data are presently available to decide whether genetic polymorphism of Hageman factor needs to be considered. It is relevant to note that human Hageman factor isolated from the plasma of a single donor gave the same cleavage patterns as seen in Figs. 5 and 6 and, therefore, if the existence of multiple forms of
Hageman factor is responsible for these observations, then the multiple forms are present in an individual.

It should also be noted that multiple forms of $\mathrm{e}$ fragment, although undifferentiated by molecular weight with the methods employed in the current studies, have been observed in alkaline polyacrylamide-gel electrophoresis (32-34) and immunoelectrophoresis in agarose (30). Cleavage of the cd fragment at site 3 , yielding a 40,000-mol wt c fragment, is observed after prolonged incubation of the plasma in glass. At $110 \mathrm{~min}$ of incubation of normal plasma in glass, $15 \%$ of the cd fragments have been converted to c. The significance of the secondary cleavage, if any, is unknown. No peak corresponding to region $\mathrm{d}(12,000 \mathrm{~mol} \mathrm{wt})$ is observed on SDS-polyacrylamide gels, presumably because this portion of the molecule is not radiolabeled.

\section{ACKNOWLEDGMENTS}

The authors wish to acknowledge the expert technical work of Alice Kleiss in the preparation of the purified plasma proteins, Gregory Beretta for the procurement of radiolabeled Hageman factor, and Lois Rude for the typing of the manuscript. Discussions with Doctors Peter Henson, Richard Ulevitch, and Bonno Bouma are also gratefully acknowledged.

This is publication number 1229 of the Department of Immunopathology, Scripps Clinic and Research Foundation, La Jolla, Calif. This work was supported by U. S. Public Health Service grant AI-07007, The Council for Tobacco Research, and contract NR207-027 from the Office of Naval Research.

\section{REFERENCES}

1. Wuepper, K. D., and C. G. Cochrane. 1972. Effect of plasma kallikrein on coagulation in vitro. Proc. Soc. Exp. Biol. Med. 141: 271-276.

2. Cochrane, C. G., S. D. Revak, B. S. Aikin, and K. D. Wuepper. 1972. The structural characteristics and activation of Hageman factor. In Inflammation: Mechanisms and Control. I. H. Lepow and P. A. Ward, editors. Academic Press, Inc., New York, 119-138.

3. Bagdasarian, A., B. Lahiri, and R. W. Colman. 1973. Origin of the high molecular weight activator of prekallikrein. J. Biol. Chem. 248: 7742-7747.

4. Cochrane, C. G., S. D. Revak, and K. D. Wuepper. 1973. Activation of Hageman factor in solid and fluid phases. A critical role of kallikrein. J. Exp. Med. 138: 1564-1583.

5. Revak, S. D., C. G. Cochrane, A. R. Johnston, and T. E. Hugli. 1974. Structural changes accompanying enzymatic activation of human Hageman factor. J. Clin. Invest. 54: 619-627.

6. Wuepper, K. D. 1972. Biochemistry and biology of components of the plasma kinin-forming system. In Inflammation: Mechanisms and Control. I. H. Lepow and P. A. Ward, editors. Academic Press, Inc., New York. 93-117.

7. Wuepper, K. D. 1973. Prekallikrein deficiency in human plasma. J. Exp. Med. 138: 1345-1355.

8. Saito, H., O. D. Ratnoff, and V. H. Donaldson. 1974. 
Defective activation of clotting, fibrinolytic, and permeability-enhancing systems in human Fletcher trait plasma. Circ. Res. 34: 641-651.

9. Weiss, A. S., J. I. Gallin, and A. P. Kaplan. 1974. Fletcher factor deficiency. A diminished rate of Hageman factor activation caused by absence of prekallikrein with abnormalities of coagulation, fibrinolysis, chemotactic activity, and kinin generation. J. Clin. Invest. 53: 622633.

10. Hathaway, W. E., L. P. Belhasen, and H. S. Hathaway. 1965. Evidence for a new plasma thromboplastin factor. I. Case report, coagulation studies and physicochemical properties. Blood. 26: 521-532.

11. Hathaway, W. E., and J. Alsever. 1970. The relation of "Fletcher factor" to factors XI and XII. Br. J. Haematol. 18: $161-169$.

12. Schiffman, S., and P. Lee. 1974. Preparation, characterization and activation of a highly purified factor XI: evidence that a hitherto unrecognized plasma activity participates in the interaction of factors XI and XII. Br. J. Haematol. 27: 101-114.

13. Colman, R. W., A. Bagdasarian, R. C. Talamo, C. F. Scott, M. Seavey, J. A. Guimaraes, J. V. Pierce, and A. P. Kaplan. 1975. Williams trait. Human kininogen deficiency with diminished levels of plasminogen proactivator and prekallikrein associated with abnormalities of the Hageman factor-dependent pathways. J. Clin. Invest. 56: 1650-1662.

14. Wuepper, K. D., D. R. Miller, and M. J. Lacombe. 1975. Flaujeac trait: deficiency of kininogen in man. Fed. Proc. 34: 859. (Abstr.)

15. Wuepper, K. D., D. R. Miller, and M. J. Lacombe. 1975. Flaujeac trait. Deficiency of human plasma kininogen. J. Clin. Invest. 56: 1663-1672.

16. Donaldson, V. H., H. I. Glueck, M. A. Miller, H. Z. Movat, and F. Habal. 1976. Kininogen deficiency in Fitzgerald trait: role of high molecular weight kininogen in clotting and fibrinolysis. J. Lab. Clin. Med. 87(2): 327-337.

17. Colman, R. W., A. Bagdasarian, R. C. Talamo, M. Seavey, C. F. Scott, and A. P. Kaplan. 1975. Williams trait: combined deficiency of plasma plasminogen proactivator, kininogen and a new procoagulant factor. Fed. Proc. 34: 859. (Abstr.)

18. Saito, H., O. D. Ratnoff, R. Waldmann, and J. P. Abraham. 1975. Fitzgerald trait. Deficiency of a hitherto unrecognized agent, Fitzgerald factor, participating in surfacemediated reactions of clotting, fibrinolysis, generation of kinins, and the property of diluted plasma enhancing vascular permeability (PF/dil). J. Clin. Invest. 55: 10821089.

19. Schiffman, S., and P. Lee. 1975. Partial purification and characterization of contact activation cofactor. J. Clin. Invest. 56: 1082-1092.

20. Griffin, J. H., and C. G. Cochrane. 1976. Involvement of high $\mathrm{M}$. W. kininogen ( $\mathrm{HK}$ ) in surface dependent reactions of Hageman factor (HF). Fed. Proc. 35: 692. (Abstr.)

21. Griffin, J. H., and C. G. Cochrane. 1976. Mechanisms for the involvement of high molecular weight kininogen in surface-dependent reactions of Hageman factor. Proc. Natl. Acad. Sci. U. S. A. 73: 2554-2558.

22. Meier, H. L., M. Webster, C. Y. Liu, R. W. Colman, and A. P. Kaplan. 1976. Enhancement of surface dependent Hageman factor activation by high molecular weight kininogen. Fed. Proc. 35: 692. (Abstr.)

23. Saito, H. 1976. Role of HMW-kininogen (Fitzgerald factor) in blood coagulation. Clin. Res. 24: 573A. (Abstr.)

24. Liu, C. Y., A. Bagdasarian, H. Meier, C. F. Scott, J. Pierce, A. P. Kaplan, and R. W. Colman. 1976. Potentiation of the action of Hageman factor fragments (HF $\left.{ }^{f}\right)$ by high molecular weight kininogen (HMWKgn). Fed. Proc. 35: 692. (Abstr.)

25. Griffin, J. H., and C. G. Cochrane. 1976. Human factor XII. In Methods in Enzymology. L. Lorand, editor. Academic Press, Inc., New York. Vol. VL part B, 56-65.

26. McConahey, P. J., and F. J. Dixon. 1966. A method of trace iodination of proteins for immunologic studies. Int. Arch. Allergy Appl. Immunol. 29: 185-189.

27. Wuepper, K. D., and C. G. Cochrane. 1972. Plasma prekallikrein: isolation, characterization, and mechanism of activation. J. Exp. Med. 135: 1-20.

28. Habal, F. M., H. Z. Movat, and C. E. Burrowes. 1974 Isolation of two functionally different kininogens from human plasma - separation from proteinase inhibitors and interaction with plasma kallikrein. Biochem. Pharmacol. 23: 2291-2303.

29. Weber, K., and M. Osborn. 1969. The reliability of molecular weight determinations by dodecyl sulfate-polyacrylamide gel electrophoresis. J. Biol. Chem. 244: 4406-4412.

30. Revak, S. D., and C. G. Cochrane. 1976. The relationship of structure and function in human Hageman factor. The association of enzymatic and binding activities with separate regions of the molecule. J. Clin. Invest. 57: 852-860.

31. Burrowes, C. E., H. Z. Movat, and M. J. Soltay. 1971. The kinin system in human plasma. VI. The action of plasmin. Proc. Soc. Exp. Biol. Med. 138: 959-966.

32. Kaplan, A. P., and K. F. Austen. 1971. A prealbumin activator of prekallikrein. II. Derivation of activators of prekallikrein from active Hageman factor by digestion with plasmin. J. Exp. Med. 133: 696-712.

33. Kaplan, A. P., and K. F. Austen. 1970. A pre-albumin activator of prekallikrein. J. Immunol. 105: 802-811.

34. Cochrane, C. G., and K. D. Wuepper. 1971. The first component of the kinin-forming system in human and rabbit plasma. Its relationship to clotting factor XII (Hageman factor). J. Exp. Med. 134: 986-1004. 